Journal of Molecular Microbiology and Biotechnology

Alvarez, M.d.F. 170

Bardy, S.L. 41

Bäumer, S. 204

Becker, A. 115

Cox, E. 155

Cranenburgh, R.M. 197

Dilworth, M.J. 133, 140

Djordjevic, M.A. 140

Ehrenreich, A. 204

Ehrenreich, P. 204

Feesche, J. 204

Fenner, B.J. 133

Glenn, A.R. 133, 140

Goddeeris, B.M. 155

Gottschalk, G. 204
Guerreiro, N. 140

Hanak, J.A.J. 197

Hebermehl, M. 148

Hefferon, K.L. 89, 109

Henne, A. 204

Herzberg, C. 204

Howieson, J.G. 133, 140

Inui, M. 182

Ishikawa, H. 212

Ishimi, K. 212

Jäger, S. 148

Jarrell, K.F. 41

Kaiser, D. 52

Kawaguchi, H. 182

Kipp, P. 109
Klug, G. 148

Lewis, K.S. 197

Liesegang, H. 204

Limberger, R.J. 30

McBridge, M.J. 63

McCarter, L.L. 18

Matsumura, M. 212

Maurer K.H 204

Medina, R. 170

Merkl, R. 204

Mimitsuka, T. 212

Minamino, T. 5

Moon, Y.S. 109

Murakami, S. 182

Muto, T. 212
Namba, K. 5

Ng, S.Y.M. 41

Nudleman, E. 52

Ogino, H. 212

Okino, S. 182

Oster, G. 72

Pasteris, S.E. 170

Quester, I. 115

Reeve, W.G. 133, 140

Rolfe, B.G. 140

Saier, M.H., Jr. 102

Schiltz, E. 148

Schultzel, M.A. 102

Sesma, F. 170

Steckel, S. 204
Strasser de Saad, A.M. 170

Stubbs, J. 140

Tiwari, R.P. 133, 140

Trachtenberg, S. 78

Veith, B. 204

Verdonck, F. 155

Vertès, A.A. 182

von Rozycki, T. 102

Wolgemuth, C.W. 72

Yasuda, M. 212

Yukawa, H. 182

\title{
Subject Index Vol. 7, 2004
}

Acid response 133, 140

Acidity 133, 140

Adhesin 155

Assembly 5

ATPase 5

Bacillus licheniformis DSM13 204

- subtilis 204

Bacterial motility 78

Baculovirus 89

Bicarbonate 102

Biofilms 52

Capping structure 5

Chaperone 5

Chitin 63

Corynebacterium glutamicum 182

Cyanobacteria 72,102

Cytophaga 63

DAP 197

Degradosome 148

DH1lacdapD 197

Diacetyl 170

Diarrhoea 155

DNA vaccine 197
Electron microscopy 78

Enterotoxigenic Escherichia coli 155

Esterase 212

Evolution 102

Expression system 109

F4 (K88) fimbriae 155

Fibrils 52

Flagellum 5, 18, 30

Flavobacterium 63

Geminivirus 109

Genome sequence 204

Glycerol kinase 170

- metabolism 170

Glycosylation 41

Industrial enzymes 204

- fermentation 204

Junctional pore complex 72

lac repressor 197

Lactate production 182

Lactobacillus rhamnosus 170

Late expression factors (LEFs) 89
Linear motors 78

Lipolytic enzyme 212

Low pH 133, 140

MAPK 109

Metabolic burden 197

Methanococcus voltae 41

Mollicutes 78

Motility 5, 18, 30, 41, 52, 63, 72

Motor proteins 78

mRNA stability 148

MucR binding site 115

Myxobacteria 72

$\mathrm{NAD}^{+} / \mathrm{NADH}$ ratio 182

Oxygen 148

- deprivation 182

Periplasm 30

Phosphate-dependent regulation 115

Photosynthesis 102

Pig 155

PilT, PilQ 52

Preflagellin peptidase 41

Proteomic analysis 140

Pseudomonas aeruginosa 212
Recombinant protein 197

Replication 109

Rhizobium meliloti 115

Rhodobacter 148

Secretins 52

Sinorhizobium 133, 140

Spirochete 50

Spiroplasma 78

Structure 30

Substrate specificity switch 5

Succinate production 182

Swarming 18

Swimming 18

Temperature 148

Transcription 89

Transcriptional fusions 133

Transport 102

Transporter 63

Type III protein export 5

- IV pili 41

Vibrio parahaemolyticus 18

Virus replication 89

\section{KARGER @ 2004 S. Karger AG, Basel}

Fax +41613061234 